\title{
IMPROVEMENT OF RESOLUTION IN APERTURE SYNTHESIS IN NEAR ZONE OF RADIATION BASED ON THE APPLICATION OF THE PRONY'S METHOD
}

\author{
O.O. Drobakhin*, O.V. Olevskyi \\ Oles Honchar Dnipro National University, Dnipro, Ukraine \\ e-mail:drobakhino@gmail.com
}

\begin{abstract}
Applicability of inverse filtering with evolution control for reconstruction of images obtained by aperture synthesis technique is considered. A horn antenna, which is moved in the transversal direction, is used in order to obtain a 2-dimensional image. Scanned structure consists of one or two similar metal plates. In the latter case the plates are coplanar and their common plane is parallel to the aperture of the antenna. Regularisation coefficients are found with the help of trial and error method. The image of a single plate was used to tune the software. The Prony's method is tested as a separate way of estimation of the distance between the plates. Both methods are tested for a range of different distances between the plates and between the plates' common plane and aperture of the antenna. It is shown that inverse filtration tends to provide better results if the scanned object is situated further from the antenna. Said method was proven to be precise enough to distinguish closely-spaced plates. The Prony's method was proven to provide reliable distance measurements if it was tuned correctly.

Keywords: aperture synthesis, inverse filtering, evolution control, Prony's method, image reconstruction, radio picture; spatial separation ability.
\end{abstract}

Received 12.11.2018; Received in revised form 15.12.2018; Accepted 21.12.2018

\section{Introduction}

Obtaining radio images in the far zone, radar characteristics is a time consuming and cost procedure due to the need to conduct measurements at large anechoic radar ranges with shielding from action of external radiation sources. Scanning in the near and intermediate zones can be carried out in conditions of a compact anechoic chamber and even a laboratory, but the difficulties of describing the peculiarities of the propagation of electromagnetic waves under conditions mentioned and the lack of reliable models are arisen. The relevance of the research of aperture synthesis is determined by the need to develop and improve the technology of aperture synthesis in the near and intermediate radiation zones to increase the resolution in radio images.

One of the key issues in receiving radio images is to improve the spatial resolution of an image. The traditional approach of improving the spatial resolution in the transverse plane is the aperture synthesis [1]. It can be considered as a matching filtration and in the far zone it is based on inverse discrete Fourier transform. In the intermediate zone it involves the use of Fresnel transform. The application of generalized spatial inverse filtering methods instead of Fresnel transformation for aperture synthesis in the near to intermediate zone is considered in [2]. Traditionally, aperture synthesis is used for data at each of the frequencies. The multifrequency approach allows for each transverse position $x$ to obtain a distance portrait of the object scanned along the longitudinal coordinate $y$. Application of recognition technologies based on neural networks can provide additional possibilities for object discrimination [3]. Some sources of noise and distortion are uncontrolled [5]. This type of external influence is considered in this paper. Spatial filtering can be done on the basis of inverse filtering algorithms with evolutionary control. Within these algorithms a number of regularization procedures can be used. The true choice of optimal regularization parameters significantly determines the effectiveness of obtaining the result.

The aim of the work was to increase the spatial separation capacity in the transverse plane, to remove the distortion received from the received picture and the noise generated by uncontrolled or unknown sources. The spectral analysis using the Prony's method [6] instead 
of DFT to identify the components that correspond to the most significant effective plane waves in the decomposition of the measured signal allows us to overcome the traditional procedure for choosing regularization parameters by choosing the number of components in the decomposition. The specific feature of using the Prony's method is the ability to cut off the noise components that are the source of distortion of the final image. In all cases of experimental investigation a scanned structure formed by one or two copper plates, the surfaces of which lay in a common plane parallel to the aperture of the antenna, were used. The Prony's method was investigated as a tool for estimating the distance between the plates and their edges. Both methods based on DFT and the Prony's method were tested for a number of values of the distance between the plates and between their common plane and aperture.

\section{The mathematical and experimental base of approach}

The first stage of image reconstruction with the help of inverse filtering is estimation of the transfer function. This function can be obtained either with the help of a mathematical model or direct measurement. Direct measurement technique allows one to avoid the construction of physical and mathematical model. On the other hand, such approach requires accurate measurement procedure and application of noise suppression techniques. A large part of such methods is computer-based.

For obtaining experimental data measuring device [7] was applied. Testing of the method was conducted with the use of the images obtained by radar scanning of straight metal plates. The faces of the plates shared a common plane which was parallel to the aperture of the antenna. All of the plates had similar height and width and were made of copper. A horn antenna was moved along the transversal axis $x$ with the step of $1 \mathrm{~mm}$. The samples of synthesized signal along longitudinal axis $y$ was obtained by application of discrete Fourier transform to multyfrequency data obtained in every point in transverse direction. An example of obtained image can be seen in Fig. 1.

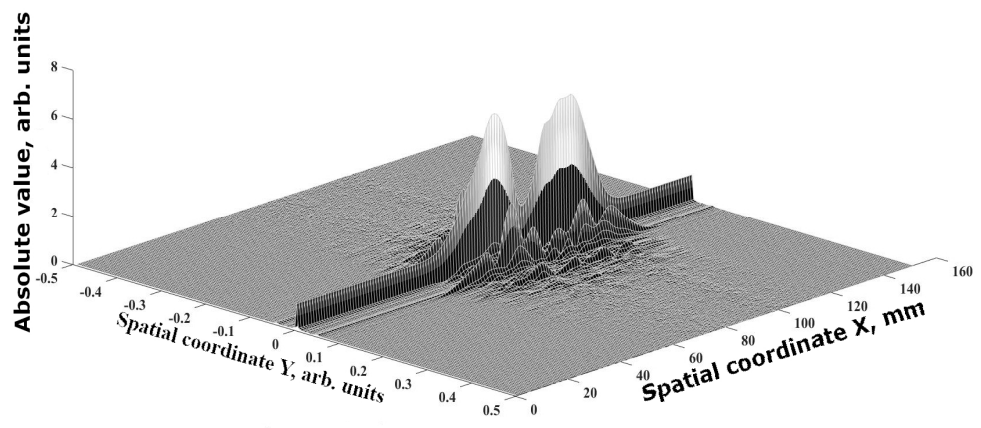

Fig. 1. An example of measured radioimage

If the initial signal spectrum $\mathrm{F}\left(y, \omega_{x}\right)$ and the output signal spectrum $\mathrm{G}\left(y, \omega_{x}\right)$ are known, we can find the transfer function as $\mathrm{H}\left(y, \omega_{x}\right)=\mathrm{G}\left(y, \omega_{x}\right) / \mathrm{F}\left(y, \omega_{x}\right)$. Obtained transfer function contains both useful data and parasitic values which appear due to presence of noise. These values can sometimes increase already existent noise to the point where the useful data becomes indistinguishable. In the current work we used the method of filtration with evolutionary control [5], which is a generalization of the inverse filtering technique. Final formula can be represented as [2] 


$$
\mathrm{S}_{\mathrm{A}}\left(y, \omega_{x}\right)=\left[\frac{\mathrm{F}\left(y, \omega_{x}\right)}{\mathrm{G}\left(y, \omega_{x}\right)+\alpha_{1}}\right]^{\sigma} \cdot\left[\frac{\left|\mathrm{H}\left(y, \omega_{x}\right)\right|^{2}}{\left|\mathrm{H}\left(y, \omega_{x}\right)\right|^{2}+\alpha_{2} \cdot \mathrm{Q}(\omega)}\right]^{\mu} \mathrm{S}\left(y, \omega_{x}\right),
$$

where $\mathrm{S}_{\mathrm{A}}\left(y, \omega_{x}\right)$ is the result of filtering procedure, $\mathrm{S}\left(y, \omega_{x}\right)$ is the Fourier image of the experimentally obtained picture after the pre-processing procedure, $\alpha_{1}, \alpha_{2} \sigma$ and $\mu$ are the regularization parameters and $\mathrm{Q}(\omega)$ is the regularization function.

Some results of application of described method is presented in Fig. 2, the method tends to work better in cases when the scanned structure is placed further away from the aperture of the antenna.

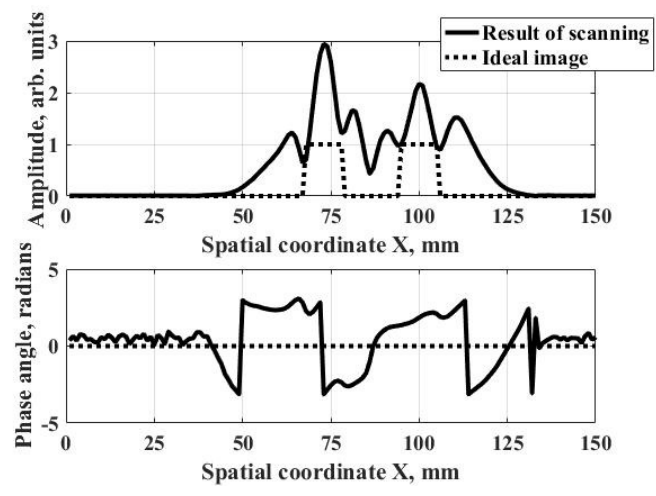

(a)
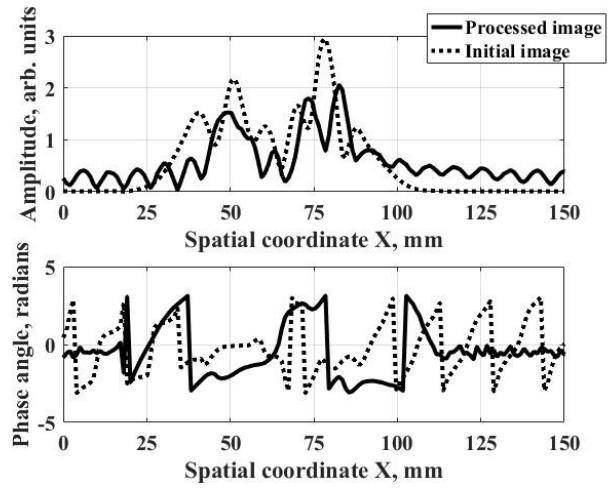

(b)

Fig. 2. Comparison between a initial image and an ideal image (a) or its processed data (b)

\section{The results of Prony's method}

The main idea of the Prony's method is to represent the Fourier image of the initial picture as a sum of exponential components with complex amplitudes and frequencies [6]. First several significant frequencies can then be interpreted as the spatial coordinates of the centers and edges of the strips. The main advantage of this method is the possibility of exclusion of insignificant components. Efficiency of the Prony's method is highly reliant on the number of the components. This is caused by the fact that while the increased number of components may lead to more accurate fitting of the spectrum shape, the stability of the approximation suffers greatly.

In order to find the optimal number of components the experiment was carried out for the closest and furthest possible distance between the plates. The obtained results were then used to estimate the distance between the plates for all available distances (table 1). An example of the processed image can be seen in Fig. 3.

Table 1

Distances obtained for plates placed $140 \mathrm{~mm}$ from the antenna with the help of the Prony's method with 40 components in the sum

\begin{tabular}{|c|c|c|c|}
\hline \multirow{2}{*}{ Real distance } & \multicolumn{2}{|c|}{ Position of the plate } & \multirow{2}{*}{$\begin{array}{c}\text { Obtained } \\
\text { distance }\end{array}$} \\
\cline { 2 - 3 } & First & Second & 14.1 \\
\hline 15 & 60.3 & 74.4 & 22.9 \\
\hline 20 & 65.6 & 88.5 & 16.89 \\
\hline 25 & 50.51 & 67.4 & 29.9 \\
\hline 30 & 49.9 & 79.8 & 33.1 \\
\hline 35 & 49.6 & 82.7 & 48.4 \\
\hline 50 & 51.3 & 99.7 & 70.5 \\
\hline 70 & 40.4 & 110.9 & \\
\hline
\end{tabular}




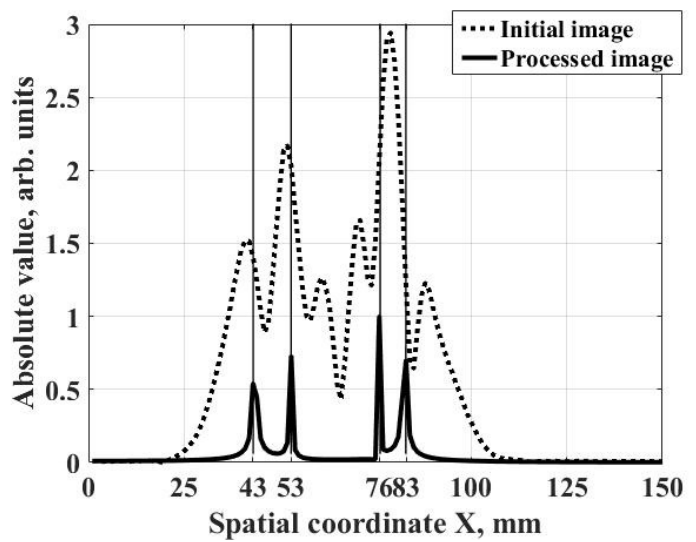

Fig. 3. Example of an image, processed with the help of the Prony's method

\section{Conclusions}

Inverse filtering with evolution control has been proven to have sufficient resolution to distinguish two metal plates positioned as closely as $15 \mathrm{~mm}$ apart under the influence of distortions caused by air and external noise sources. It was shown that the quality of image recovery increases if the scanned structure is positioned further from the aperture. The Prony's method was proven to be able to reliably estimate distance between the objects regardless of distance between them.

\section{References}

1. Osumi, N. Microwave holographic imaging of underground objects / N. Osumi, K. Ueno // IEEE Transactions on Antennas and Propagation - Vol. 33, No. 2. - pp. 152 - 159. doi: 10.1109/TAP.1985.1143559

2. Bulany, M.F. Multifrequency microwave images with inverse synthetic aperture in the intermediate zone of radiation / M.F. Bulany, O.O. Drobakhin, G.G. Sherstyuk // Visnyk Dnipropetrovs'kogo universytetu. Fizyka. Radioelectronika. - 2014, Issue 21. - Vol. 22, No 1. - P. $133-138$.

3. Drobakhin, O.O. Recognition multifrequency microwave images of simple objects behind dielectric wall using neural networks and correlation technique / O.O. Drobakhin, G.G. Sherstyuk // Conference proceedings of 2013 XVIIIth International Seminar/Workshop on Direct and Inverse Problems of Electromagnetic and Acoustic Wave Theory (DIPED), - Lviv, 2013. - P. 133 - 136. doi: 10.1109/MSMW.2013.6622099

4. Vasilenko, G.I. Image restoration / G.I. Vasilenko, A.M. Taratorin. - Moscow: Radio i svyaz', 1986. $-302 \mathrm{p}$.

5. Andreev, M.V. The Prony's method realization in the presence of pulse noise / M.V. Andreev, O.O. Drobakhin, S.G. Lebedev // Conference proceedings of 2012 Int. Conf on Mathematical Methods in Electromagnetic Theory, - Kyiv, 2012. - P. 161 - 163.

6. Alekseev, V.V. Multifrequency Near-Zone Radar of 6-mm Wave Range with Combination of Pulse Synthesis and Transversal Scanning / V.V.Alekseev, M.V.Andreev, O.O. Drobakhin, Ye.V. Kondrat'ev, D.Yu. Saltykov // Telecommunications and Radio Engineering - Vol. 66, No. 10. - P. 855 - 861. doi: 10.1615/TelecomRadEng.v66.i10.10

7. Lyasota, D. Recognition of conductive objects based on the characteristics of reflected electromagnetic wave / D. Lyasota, V.M. Morozov, V.I. Magro // Radioelectronics and Communications Systems - 2016. - Vol. 59, No. 7. - P. 293 - 300. 